УДК 581.4+582.949.2(517.3)

\title{
Fruit morphology of some species of the Lamiaceae in the flora of Mongolia
}

\author{
Bazarragchaa Badamtsetseg \\ Mongolian Museum of Natural History, Sambuu street, Chingeltei district, Ulaanbaatar-46, Mongolia. \\ E-mail: batamtsetseg@yahoo.com
}

Key words: nutlets, size, shape, surface, ornamentation, color, Ajugoideae, Scutellarioideae, Nepetoideae, Lamioideae.

Summary. Nutlet morphology in the family Lamiaceae was examined using scanning electron microscopy (SEM) to identify distinct characters for 20 species belonging to 12 genera in the subfamilies: Ajugoideae, Scutellarioideae, Nepetoideae and Lamioideae. Nutlet morphology in the family Lamiaceae showed considerable variation in size, color (light orange-yellow, light nut-brown, pale grey, black and all that), shape (subglobose, obovoid, subobovoid, elliptic), surface ornamentation (type I, type II), nutlet apex (rounded, triangle) and base (truncate, acute, rounded). The described types have been collected in the territory of Mongolia. In Lamiaceae two main types can be recognized based on surface ornamentation: sculptured - type I (including 8 species) and smooth - type II (including 12 species). Within these types subtypes were recognized. Type I: Sculptured nutlets are characterized by having oblong, coneshaped and ellipsoid tubercles occuring on all surfaces. The tubercles densely covered with longish mastoid white color in apex and covered by white hairs, with white mastoids under hairs. Five subtypes can be recognized based on the shape of the tubercles: colliculate-reticulate, wrinkled-colliculate-tuberculate, wrinkled-tuberculate, colliculatetuberculate, hairy. Type II: The smooth nutlets may be divided in 4 subtypes: irregularly reticulate, reticulate, undulate, wrinkled-colliculate.

\section{Морфология плодов некоторых видов Lamiaceae флоры Монголии}

\author{
Базаррагчаа Бадамцэцэг
}

Монгольский музей естественной истории, ул. Самбуу, район Чингэлтэй, Улаанбаатар-46, Монголия

Ключевые слова: эремы, морфологические признаки, скульптура поверхности, Ajugoideae, Scutellarioideae, Nepetoideae, Lamioideae.

\begin{abstract}
Аннотация. Приведены результаты исследования морфологии эремов 20 видов губоцветных (Lamiaceae), выполненного при помощи сканирующей электронной микроскопии (CЭM). Дана характеристика плодов представителей 12 родов подсемейств Ajugoideae, Scutellarioideae, Nepetoideae, Lamioideae. Эремы имеют значительные различия в размерах, окраске (светло-оранжево-желтые, светло-коричневые, светло-серые, черные), форме (шаровидные, обратнояйцевидные, яйцевидные, эллипсовидные), скульптуре поверхности (тип I, тип II), форме верхушки (округлая, треугольная) и основания (обрубленное, острое или округлое). Описанные виды собраны на территории Монголии. В семействе Lamiaceae два основных типа могут быть признаны на основе поверхностного орнамента: тип I - рельефный (8 видов), тип II - гладкий (12 видов). Внутри этих типов можно выделить подтипы. В первом типе могут быть признаны пять подтипов на основании формы бугорков: бородавчато-сетчатых, морщинисто-бородавчато-бугорчатых, морщинисто-бугорчатых, бородавчато-бугорчатых, опушенных. Тип II с гладкими эремами могут быть разделены на 4 подтипа: с неравномерносетчатыми бугорками, сетчатыми, волнистыми, морщинисто-бородавчатыми.
\end{abstract}




\section{Introduction}

The Mint family (Lamiaceae) is one of the largest Angiosperm families, comprising approximately 240 genera and more than 7000 infrageneric taxa distributed throughout the world and economically very important (Harley, 2003). In the flora of Mongolia Lamiaceae is one of the ten largest plant families represented by 89 taxa belonging to 24 genera and four subfamilies (Badamtsetseg, 2010).

Fruit of Lamiaceae is coenobium, thereof named tetraeremum based on nutlets number, and 18 variants based on nutlets forma, 22 variants based on nutlets surface features (Artyushenko, Fedorov, 1986). Fruit of Lamiaceae is uncluded in coenobium group by the fruit classification and has different forms and surface features (Levina, 1987).

Ultrastructure features of nutlets surface can be used in the taxonomy of the genus Nepeta, and selected group of genera represents the branches of the tribe Nepeteae differentiation which took place in the early stages of its evolution (Budantsev, 1993a, b). Nutlet morphology in the Lamiaceae has been proved useful in varying degrees at different levels of the taxonomic hierarchy (Budantsev, Lobova, 1997). Nutlet characters within the genus Nepeta has a taxonomic significance (Kaya, Dirmenci, 2008).

\section{Material and methods}

This study is based on nutlets taken from herbarium specimens, mostly from UBU, ALTB and MMNH (Mongolian Museum of Natural History), and original collections made during expeditions to Khentii, Khubsugul, Uvurkhangai, Tov and Dornogovi provinces. For SEM studies nutlets were coated with carbon using a Hitachi E-1010 ion sputter, observed in a Hitachi S-3400N and photographed. All photomicrographs were made at the department of SEM laboratory, National Science Museum, city Deajeon of South Korea. Nutlet color was defined using color chart. Measurements of nutlet were carried out by reiteration 15-20 times under a microscope Olympus BX 40. The nutlet shape and surface ornamentation were studied following Vit Bojnansky, Agata Fargasova (2007), seed morphology descriptions were carried out with the method Joongku Lee et al. (2009).

\section{Results}

We studied nutlet morphology of 20 species belonging to 12 genera of Lamiaceae found in Mongolia. Morphological variation of the size, shape, color, surface type, hilum lay of the seeds in Lamiaceae is described below. We wrote seed morphology descriptions for all these 20 species.

Nutlet color varies from light orange-yellow, light nut-brown, medium buff, pale grey, grey nut-brown, light brown, dark brown, red-brown to black. Nutlet shapes vary from obovoid, suboboviod to elliptic, subglobose. Nutlet apex varies from rounded to triangle and base varies from truncate, acute to rounded. Nutlet surface varies from sculptured and smooth to glabrous and covered by hairs.

In Lamiaceae two main types can be recognized based on surface ornamentation: sculptured - type I, and smooth - type II. Within these types subtypes can be recognized.

TYPE I: Sculptured nutlets are characterized by having oblong, cone-shaped and ellipsoid tubercles occur on all surfaces. The tubercles densely covered with longish mastoid white color in apex and covered by white hairs, with white mastoids under hairs. Five subtypes can be recognized based on the shape of the tubercles:

Colliculate-reticulate (fig. 1-1 a, b, c, d): Oblong shaped tubercles covered on all surfaces of lateral view. Anticlinal walls (AW) slightly raised, periclinal walls (EPW) slightly depressed, finegranular (Amethystea coerulea L.).

Wrinkled-colliculate-tuberculate (fig. 1-2 a, b, c, d; 1-6 a, b, c, d) covered on all surfaces tuberculate with mastoids between tubercles (Scutellaria galericulata L.), and tubercles ellipsoid, apex truncate, densely covered by tubercles. Tubercles oblong shaped (Scutellaria tuvensis Juz.).

Wrinkled-tuberculate (fig. 1-3 a, b, c, d) covered on all surfaces by tubercles cone-shaped, apex truncate. The tubercles densely covered with longish mastoid white color in apex (Scutellaria baicalensis Georg.).

Colliculate-tuberculate (fig. 1-4 a, b, c, d) covered on all surfaces by tubercles ellipsoid, apex rounded, densely covered with tubercles. The tubercles have shortish mastoids white in apex ( $\mathrm{Scu}$ tellaria scordifolia Fisch. ex Scrank.).

Hairy (fig. 1-5 a, b, c, d; 1-7 a, b, c, d; 1-8 a, b, c, d) covered on all surfaces by hairs and hairs with white mastoids under hairs (Scutellaria grandiflora Sims., S. sieversii Bunge., S. supina L.).

TYPE II: The smooth nutlets may be divided in 4 subtypes.

Irregularly reticulate (fig. 2-10 a, b, c, d; 2-13 a, b, c, d; 3-17 a, b, c, d; 3-18 a, b, c, d) - has a raised network on the surface, often cells formed indistinctly shaped. Anticlinal walls raised, cells 
indistinctly shaped, periclinal walls flat or slightly depressed (Schizonepeta annua L., Mentha arvensis L., Panzerina lanata (L.) Sojak., Lagochilus ilicifolius Bunge.).

Reticulate (fig. 2-9 a, b, c, d; 3-14 a, b, c, d; 3-15 a, b, c, d; 3-19 a, b, c, d; 3-20 a, b, c, d) - has a raised network on the surface, often cells flat shaped. Anticlinal walls raised or slightly depressed, cells tetra, penta- or hexagonal, periclinal walls slightly depressed or slightly raised (Lophanthus chinensis (Rafin., ) Benth., Galeopsis bifida Boenn., Leonurus sibiricus L., Lagopsis marrubiastrum (Steph.) Ik.Gal. Stachys palustris L.).

Undulate (fig. 2-11 a, b, c, d; 3-16 a, b, c, d) wavy, but not so deeply or as pronounced as sinuate.

Anticlinal walls raised indistinctly shaped or slightly raised, periclinal walls striate-furrowed or wrinkling pronounced as sinuate (Schizonepeta multifida Brig., Leonurus deminutus Krecz.).

Wrinkled-colliculate (fig. 2-12 a, b, c, d) - with a surface pattern of regular distinct rounded hillocks, each usually formed a wrinkling. Anticlinal walls depressed, periclinal walls low raised, tubercles indistinctly shaped and strongly wrinkled (Thymus gobicus Tschern.).

Subfamily Ajugoideae Luerss.

Amethystea coerulea L.: "River Shuus, BayanAdarga soum, Khentii province, $985 \mathrm{~m}, \mathrm{~N} 48.36231^{\circ}$, E111.24297 22 VIII 2009. B. Badamtsetseg" (MMNH).

Nutlets length $1.51 \mathrm{~mm}$, width $0.99 \mathrm{~mm}$, obovoid, dorsal side convex, ventral side mostly flat, forms the hilum, light nut-brown. Surface mostly smooth, glabrous, colliculate-reticulate: anticlinal walls (AW) slightly raised, periclinal walls (EPW) slightly depressed, fine-granular (fig. 1-1 a, b, c, d). Type I-1.

Subfamily Scutellarioideae Prantl.

Scutellaria galericulata L.: "River Onon, Bayan-Adarga soum, Khentii province, N48.42445, E111.2315.27 VI 2007. B. Badamtsetseg"(MMNH).

Nutlets length $1.52 \mathrm{~mm}$, width $1.12 \mathrm{~mm}$, subobovoid, light orange-yellow in color, hilum small, almost in center of ventral side. Surface rough, glabrous, wrinkled-colliculate-tuberculate with globes between tubercles. Tubercles ellipsoid, apex rounded. The tubercles have indistinct shape. Anticlinal walls deeply depressed, periclinal walls raised (fig. 1-2 a, b, c, d). Type I-2.

Scutellaria baicalensis Georg.: "River Shuus, Bayan-Adarga soum, Khentii province, $980 \mathrm{~m}$,
N48.3623.1 ${ }^{\circ}$, E111.2429.7․ 22 VIII 2008. B. Badamtsetseg" (MMNH).

Nutlets length $1.28 \mathrm{~mm}$, width $1.05 \mathrm{~mm}$, subobovoid, dull, dark in color. Surface rough, glabrous, wrinkled-tuberculate, tubercles coneshaped, apex truncate. The tubercles densely covered with longish mastoid white color in apex. Anticlinal walls deeply depressed, periclinal walls raised (fig. 1-3 a, b, c, d). Type I-3.

Scutellaria scordifolia Fisch. ex Scrank.: "River Toil, Jargalant soum, Khuvsugul province, N48.29584, E99.22420. 18 VIII 2006. B. Badamtsetseg" (MMNH).

Nutlets length $1.23 \mathrm{~mm}$, width $1 \mathrm{~mm}$, subobovoid, dull, light nut-brown in color. Surface rough, glabrous, colliculate-tuberculate. Tubercles ellipsoid, apex rounded, densely covered with tubercles. The tubercles have shortish mastoids white in apex. Anticlinal walls deeply depressed, periclinal walls raised (fig. 1-4 a, b, c, d). Type I-4.

Scutellaria grandiflora Sims.: "Mountain Teshir Herbei, western south from Sagsai soum, BayanUlgii province, N48.57, E 89.30. 01 VIII 2001. R. V. Kamelin, A. I. Shmakov et al." (ALTB).

Nutlets subobovoid, dull, pale grey. Surface rough, covered by white hairs, with white mastoids under hairs (fig. 1-5 a, b, c, d). Type I-4.

Scutellaria tuvensis Juz.: "Tuva, distr. Ovjur, prope pag. Ak-Czira, declivitas australis jugi Tannu-Ola Orientalis in schistosis. 13 VIII 1973. S. Timokhina M. Sakovicz" (ALTB).

Nutlets length $1.25 \mathrm{~mm}$, width $0.96 \mathrm{~mm}$, subobovoid, dull, black. Surface rough, glabrous, wrinkled-colliculate-tuberculate. Tubercles ellipsoid, apex truncate, densely covered tubercles. Tubercles oblong shaped. Anticlinal walls deeply depressed, periclinal walls raised (fig. 1-6 a, b, c, d). Type I-2.

Scutellaria sieversii Bunge: "Kazakhstan, distr. Taldi-Kurgan, Dzungar Alatau, west coast of river Middle Tentek. 21 VII 1991. A. I. Shmakov, A. L. Ebel" (ALTB).

Nutlets length $1.38 \mathrm{~mm}$, width $1.08 \mathrm{~mm}$, subobovoid, dull, pale grey in color. Surface rough, densely covered with white hairs, hairy (fig. 1-7 a, b, c, d). Type I-4.

Scutellaria supina L.: "River Tsagaan us, Bayan-Ulgii province, N48.57, E88.03. 30 VII 2001. R. V. Kamelin, A. I. Shmakov" (ALTB).

Nutlets length $1.24 \mathrm{~mm}$, width $0.98 \mathrm{~mm}$, subobovoid, dull, pale grey in color. Surface rough, densely covered with white hairs, hairy (fig. 1-8 a, b, c, d). Type I-4. 

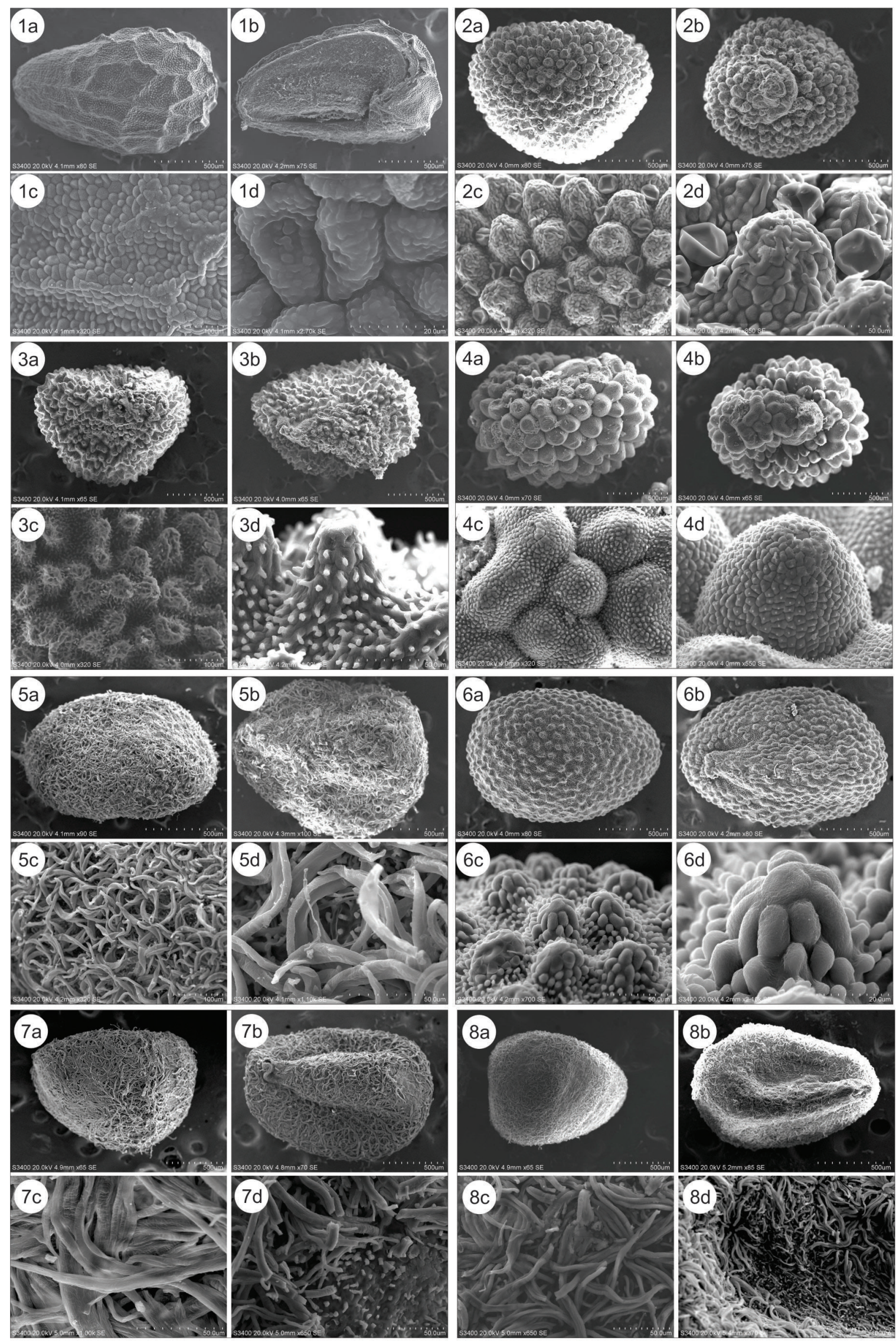

Fig. 1. SEM micrographs of nutlets of subfamily Ajugoideae and Scutellarioideae: 1 - Amethystea coerulea, 2 Scutellaria galericulata, 3 - S. baicalensis, 4 - S. scordifolia, 5 - S. grandiflora, 6 - S. tuvensis, 7 - S. sieversii, 8 S. supine, a - lateral view, $\mathrm{b}$ - ventral view, c-d - ornamentation of surface. 


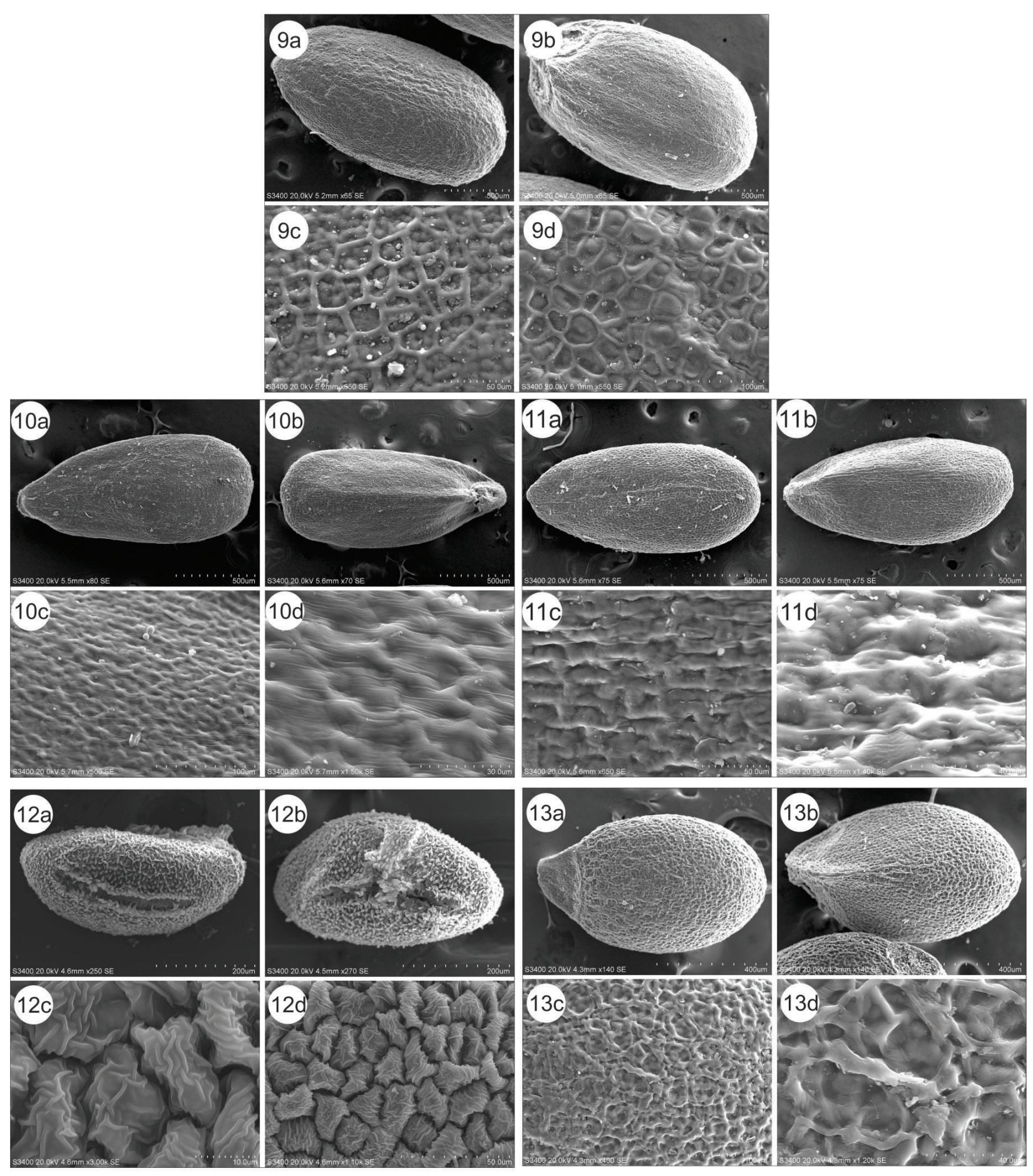

Fig. 2. SEM micrographs of nutlets of subfamily Nepetoideae: 9 - Lophanthus chinensis, 10 - Schizonepeta annua, 11 - Sch. multifida, 12 - Thymus gobicus, 13 - Mentha arvensis, a - lateral view, b - ventral view, c- $\mathrm{d}$ - ornamentation of surface.

\section{Subfamily Nepetoideae Burnett}

Lophanthus chinensis (Rafin.) Benth.: "Mountain Toil Yargait. Jargalant soum, Khuvsugul province, N48.29584 ${ }^{\circ}$ E99.22437 19 VII 2006. B. Badamtsetseg" (MMNH).

Nutlets length $1.87 \mathrm{~mm}$, width $1 \mathrm{~mm}$, obovoid, apex rounded, base almost rounded, light nut-brown in color. Surface smooth, glabrous, reticulate. Anticlinal walls raised, cells tetra-pentagonal, periclinal walls slightly depressed, smooth (fig. 2-9 a, b, c, d). Type II-2.

Schizonepeta annua L.: "Mountain Arts Bogd, Bogd soum, Uvurkhangai province, N44.1431 ${ }^{\circ}$, E102.2330 . 28 VII 2011. B. Badamtsetseg" (MMNH). 

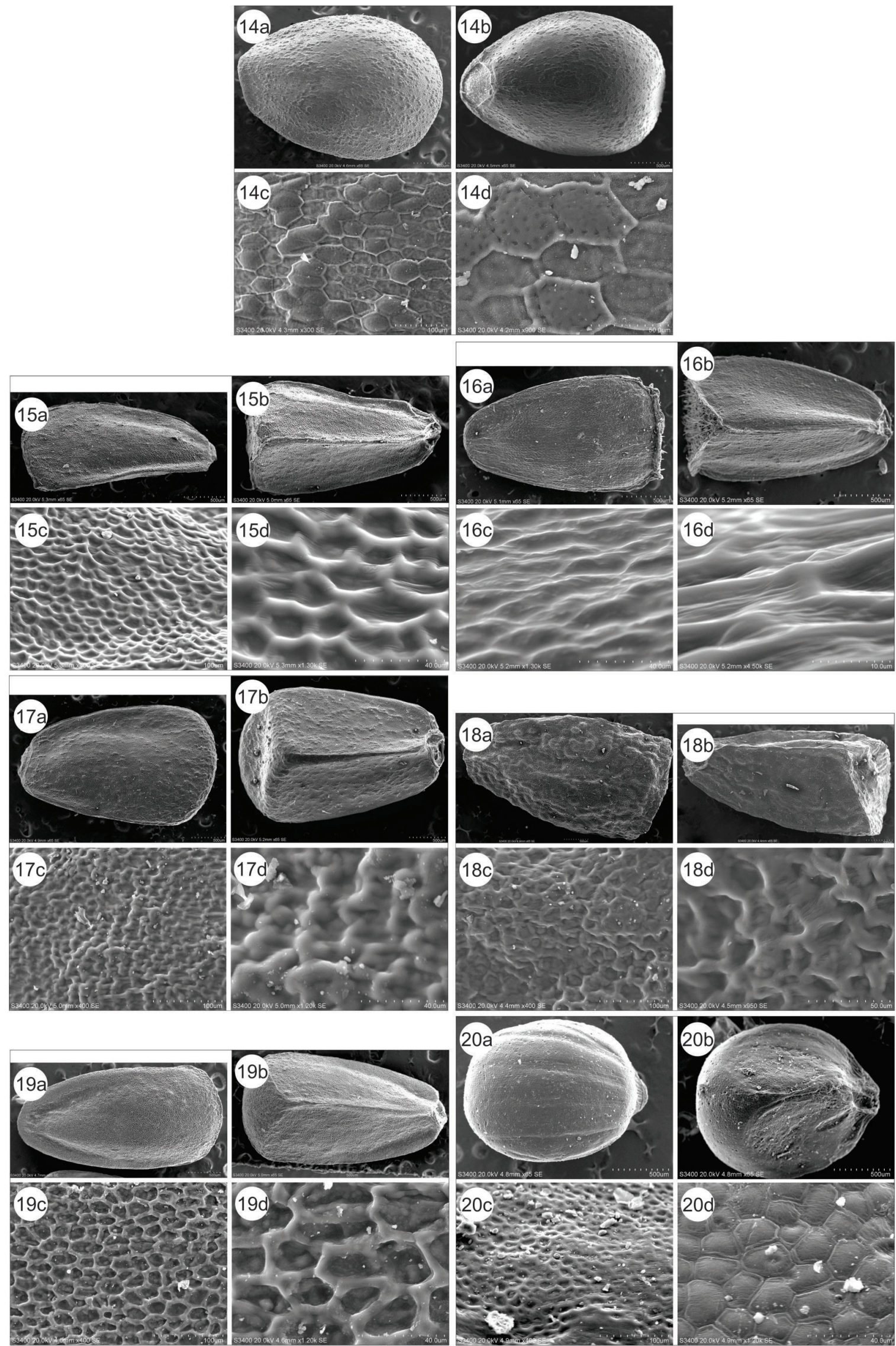

Fig. 3. SEM micrographs of nutlets of subfamily Lamioideae: 14 - Galeopsis bifida, 15 - Leonurus sibiricus, 16 - L. deminutus, 17 - Panzerina lanata, 18 - Lagochilus ilicifolius, 19 - Lagopsis marrubiastrum, 20 - Stachys palustris, $\mathrm{a}$ - lateral view, $\mathrm{b}$ - ventral view, c-d - ornamentation of surface. 
Nutlets length $1.70 \mathrm{~mm}$, width $0.70 \mathrm{~mm}$, elliptic, with 3 flattened faces, apex rounded, dark brown in color. Surface smooth, glabrous, lateral surface irregularly reticulate. Anticlinal walls raised, cells indistinctly shaped, periclinal walls depressed by little area. Ventral surface undulate (fig. 2-10 a, b, c, d). Type II-1.

Schizonepeta multifida Briq.: "River Toil, Jargalant soum, Khuvsugul province, N48.29584, E99.22437. 19 VIII 2006. B. Badamtsetseg" (MMNH).

Nutlets length $1.40 \mathrm{~mm}$, width $0.68 \mathrm{~mm}$, elliptic, apex rounded, base almost rounded, slightly flattened, grey nut-brown in color. Surface smooth, glabrous, undulate. Anticlinal walls raised indistinctly shaped, periclinal walls striate-furrowed (fig. 2-11 a, b, c, d). Type II-3.

Thymus gobicus Tschern.: "River Tuul, Erdene soum, Tov province, $1543 \mathrm{~m}, \mathrm{~N} 48.06436$, E107.51347. 20 VII 2006. B. Badamtsetseg (MMNH)".

Nutlets length $0.75 \mathrm{~mm}$, width $0.65 \mathrm{~mm}$, subobovoid, slightly curved, apex and base rounded, red-brown in color. Surface smooth, glabrous, wrinkled-colliculate. Anticlinal walls depressed, periclinal walls low raised tubercles, tubercles indistinctly shaped and strongly wrinkled (fig. 2-12 a, b, c, d). Type II-4.

Mentha arvensis L.: "River Onon, Dadal soum, Khentii province, $\mathrm{N} 48.47407^{\circ}, \mathrm{E} 111.37333^{\circ} .22$ VIII 2009. B. Badamtsetseg" (MMNH).

Nutlets length $0.82 \mathrm{~mm}$, width $0.58 \mathrm{~mm}$, obovoid, 3 flattened faces, apex rounded, light brown in color. Surface smooth, glabrous, irregularly reticulate. Anticlinal walls raised cells indistinctly shaped, periclinal walls flat depressed (fig. 2-13 a, b, c, d). Type II-1.

\section{Subfamily Lamioideae Harley}

Galeopsis bifida Boenn.: "Gorkhi-Terelj national park, Sergelen soum, Tov province. 22 VII 2004. B. Badamtsetseg" (MMNH).

Nutlets length $2.96 \mathrm{~mm}$, width $2.08 \mathrm{~mm}$, obovoid, base truncate, apex rounded, light nut-brown with black spots. Surface smooth, glabrous, reticulate, anticlinal walls testa cell penta-hexagonal, periclinal walls slightly depressed, and raised in the spots (fig. 3-14 a, b, c, d). Type II-2.

Leonurus sibiricus L.: "10 km northern from city Ondorkhaan, Khentii province. 21 VIII 2009. B. Badamtsetseg" (MMNH).

Nutlets length $2.12 \mathrm{~mm}$, width $1.07 \mathrm{~mm}$, elliptic with 3 flattened faces, apex and base truncate, light nut-brown in color. Surface smooth, glabrous, reticulate, anticlinal walls strongly raised, testa cells pentagonal, periclinal walls depressed, smooth or slightly wrinkled (fig. 3-15 a, b, c, d). Type II-2.

Leonurus deminutus Krecz.: "Near of Dresden camp, river Kherlen, Mungunmorit soum, Tov province, $1645 \mathrm{~m}, \mathrm{~N} 47.49916$, E107.37457. $01 \mathrm{VII}$ 2005. B. Badamtsetseg" (MMNH).

Nutlets length $2.12 \mathrm{~mm}$, width $1.07 \mathrm{~mm}$, obovoid with 3 flattened faces, apex and base truncate, medium buff in color. Surface smooth, glabrous, only apex hairy, undulate. Anticlinal walls slightly raised, testa cells indistinctly shaped, periclinal walls wrinkling pronounced as sinuate (fig. 3-16 a, b, c, d). Type II-3.

Panzerina lanata (L.) Soják.: "Near of Dresden camp, river Kherlen, Mungunmorit soum, Tov province, $1645 \mathrm{~m}, \mathrm{~N} 47.49916$, E107.37457. 04.VIII 2009. B. Badamtsetseg" (MMNH).

Nutlets length $3.11 \mathrm{~mm}$, width $1.85 \mathrm{~mm}$, obovoid with 3 angular faces, dark brown in color. Surface smooth with granules, glabrous, irregularly reticulate. Antilinal walls strongly raised, testa cells indistinctly shaped. Periclinal walls depressed, smooth (fig. 3-17 a, b, c, d). Type II-1.

Lagochilus ilicifolius Bunge: "Mountain Ergeliin zoo, Hatanbulag soum, Dornogovi province, N43.1151, E109.0618. 22 VII 2011. B. Badamtsetseg" (MMNH).

Nutlets length $3.3 \mathrm{~mm}$, width $2.01 \mathrm{~mm}$, elliptic with 3 flattened faces, base truncate, black in color. Surface rough, glabrous, irregularly reticulate. Anticlinal walls raised, testa cells indistinctly shaped. Periclinal walls flat depressed (fig. 3-18 a, b, c, d). Type II-1.

Lagopsis marrubiastrum (Steph.) Ik.-Gal.: "Mountain Gurvanbulag, Gurvanbulag soum, Bayankhongor province. N47.15, E98.15. 07 VII 1977. E. Ganbold" (UBU).

Nutlets length $2.1 \mathrm{~mm}$, width $1.05 \mathrm{~mm}$, obovoid with 3 angular faces, glabrous, pale grey, surface reticulate. Anticlinal walls strongly raised, testa cells tetra-pentagonal, periclinal flat depressed (fig. 3-19 a, b, c, d). Type II-2.

Stachys palustris L.: "River Onon, Dadal soum, Khentii province, $\mathrm{N} 48.47407^{\circ}, \mathrm{E} 111.37337^{\circ} .22$ VII 2009. B. Badamtsetseg" (MMNH).

Nutlets length $1.25 \mathrm{~mm}$, width $1.01 \mathrm{~mm}$, subglobose, brown. Surface smooth, glabrous, reticulate. Anticlinal walls capillary, slightly depressed, testa cells penta-hexagonal, periclinal walls slightly raised by penta-hexogonal granules with bordering (fig. 3-20 a, b, c, d). Type II-2. 


\section{Conclusions}

We studied the seed morphology of 20 species belonging to 12 genera of family Lamiaceae found in Mongolia and wrote descriptions for them.

Nutlets have obovoid, suboboviod, elliptic, subglobose shapes; surface could be rough and smooth, glabrous and covered by hairs; light orange-yellow, light nut-brown, medium buff, pale grey, grey nut-brown, light brown, dark brown, redbrown, black in color.

The surface ornamentation of nutlets of Lamiaceae was classified into 2 types, 9 subtypes: colliculate-reticulate (1 species of subfam. Ajugoideae), wrinkled-colliculate-tuberculate, wrinkled-tuberculate, colliculate-tuberculate, hairy (7 species of subfam. Scutellarioideae), irregularly reticulate, reticulate, undulate, wrinkled-colliculate
(5 species of subfam. Nepetoideae and 7 species of subfam. Lamioideae).

We consider that nutlet morphology has varying degrees at subfamily, genera and species levels of the taxonomic hierarchy. Fruit characters are consistent at generic or species level and therefore it is demanded to study nutlet characters of other genus and species of family Lamiaceae.

\section{Acknowledgements}

The authors thank Lee Sang Myoung and Peak Woon Kee from the Biological Laboratory of National Science Museum for helping with scanning electron microscopy and especially the doctor, entomologist Oh Seung Ho and collective staff of South-Siberian Botanical Garden (ALTB), Altai for managing herbarium specimens.

\section{REFERENCES}

Artyushenko Z. T., Fedorov Al. A. (1986) Organocraphia illustrata plantarum vascularum. Fruit. Nauka, Leningrad, 392 pp. [In Russian]. (Артюшенко 3. T., Федоров Ал. А. Атлас по описательной морфологии высших растений. Плод. Ленинград: Наука, 1986. 392 с.).

Badamtsetseg B. (2010) The review systematic of the Mint family (Lamiaceae Lindl.) in Mongolia // Ecological consequences of biosphere processes in the ecotone zone of Southern Siberia and Central Asia: Proceedings of the International Conference. Vol. 2. (Ulaanbaatar (Mongolia), September 6-8, 2010). Bembi san, Ulaanbaatar, 203206 pp. [In Mongolian]. (Бадамцэцэг Б. Монгол орны Уруулцэцэгтний овгийн ангилалзүйн тойм (Lamiaceae Lindl.) // Төв Ази, Өмнөд Сибирийн шилжилтийн экобүсийн шим мандал дахь экологийн үр дагавар: Олон улсын хурлын хураангуй, 2. Улаанбаатар: Бэмби сан, 2010. С. 203-206).

Bojnansky V., Fargasova A. (2007) Atlas of seeds and fruits of Central and East-European Flora: The Carpathian Mountains Region. Springer, London, 1046 pp.

Budantsev A. L. (1993a) Ultrastructural features of fruit surface in genus Nepeta (Lamiaceae) // Bot. Zhurn. (Moscow, St. Peterburg) 78(4): 80-87 [In Russian]. (Буданцев А. Л. Особенности ультраструктуры поверхности плодов видов рода Nepeta (Lamiaceae) // Бот. журн., 1993. Т. 78, № 4. C. 80-87).

Budantsev A. L. (1993b) The ultrastructural features of fruit surface in some genera of the tribe Nepeteae (Lamiaceae) // Bot. Zhurn. (Moscow, St. Peterburg) 78(5): 100-108 [In Russian]. (Буданцев А.Л. Особенности ультраструктуры поверхности плодов некоторых родов трибы Nepeteae (Lamiaceae) // Бот. журн., 1993. Т. 78, № 5. C. 100-108).

Budantsev A. L., Lobova T. A. (1997) Fruit morphology, anatomy and taxonomy of tribe Nepeteae (Labiatae) // Edinburgh J. Bot. 54, 2: 183-216.

Harley R. M. (2003) Validation of the name Lamioideae (Labiatae) // Kew Bull., 58, 3: 765-766.

Kaya, Dirmenci (2008) Nutlet surface micromorphology of the genus Nepeta L. (Lamiaceae) in Turkey // Turk. J. Bot. 32: 103-112.

Lee J., Kim J.-H., Lee S. M., Park S.-H. Ali M. A., Kim J., Lee C., Kim G.. (2009) Seeds of wild plants of Korea. Daejeon, 383 pp.

Levina R. E. (1987) Morphologiya i ecologiya plodov [Morphology and ecology of fruits]. Nauka, Leningrad, 158 pp. [In Russian]. (Левина P. E. Морфология и экология плодов. Л.: Изд-во «Наука», 1987. 158 с.). 